\title{
Philosophiques
}

\section{John Leslie, Infinite Minds, Oxford, Oxford University Press, 2001, 234 pages.}

\section{Paul Franceschi}

Volume 30, numéro 2, automne 2003

URI : https://id.erudit.org/iderudit/008655ar

DOI : https://doi.org/10.7202/008655ar

Aller au sommaire du numéro

Éditeur(s)

Société de philosophie du Québec

ISSN

0316-2923 (imprimé)

1492-1391 (numérique)

Découvrir la revue

Citer ce compte rendu

Franceschi, P. (2003). Compte rendu de [John Leslie, Infinite Minds, Oxford, Oxford University Press, 2001, 234 pages.] Philosophiques, 30(2), 453-455.

https://doi.org/10.7202/008655ar d'utilisation que vous pouvez consulter en ligne.

https://apropos.erudit.org/fr/usagers/politique-dutilisation/ 
de l'ego, et c'est dans cette optique qu'il recourt à la philosophie biranienne - on est loin de Merleau-Ponty et consorts. Bien sûr, l'auteur n'a pas à prendre le détour henrien. Cependant, on ne peut que le regretter quand on connaît la perspicacité de l'auteur et la minutie de son travail.

MICHEL RATTÉ

Université du Québec à Trois-Rivières

\section{John Leslie, Infinite Minds, Oxford, Oxford University Press, 2001, 234 pages.}

Infinite Minds constitue le quatrième ouvrage de John Leslie, qui fait suite à Value and Existence (1979), Universes (1989) et The End of the World (1996). Infinite Minds présente un contenu très riche et couvre un nombre de sujets particulièrement variés. Parmi ces derniers, on peut citer notamment: l'omniscience, le problème du Mal, l'argument du bon réglage (fine-tuning argument), les effets de sélection observationnels, l'identité des indiscernables, le temps, l'infini, la nature de la conscience.

L'ouvrage se situe clairement dans le domaine de la philosophie spéculative. Et Leslie s'y montre essentiellement préoccupé par des considérations non pas de démonstration rigoureuse, mais de plausibilité et de cohérence. Ainsi, il n'hésite pas à attribuer parfois une probabilité assez faible à certaines affirmations.

Un certain nombre de lecteurs pourront être rebutés dès le début par l'affirmation contraire à l'intuition selon laquelle les galaxies, les planètes, les animaux, mais aussi chacun d'entre nous et les objets qui nous environnent, constituent des structures au sein de pensées divines. On peut penser que c'est une telle affirmation qui a motivé le commentaire placé sur la couverture du livre par un lecteur d'Oxford University Press selon lequel il peut être difficile de croire que l'univers est tel que le décrit l'auteur. Cela a été aussi ma réaction première. Mais si certains lecteurs devaient en tirer une conclusion hâtive, ils manqueraient alors, je pense, ce qui constitue le trésor caché du livre. Car Infinite Minds ressemble à un temple somptueux dont l'accès, toutefois, se trouve dissimulé par un portail d'aspect peu engageant. Ceux qui ne franchiront pas la porte, rebutés par l'aspect de cette dernière, n'auront pas l'occasion de contempler les trésors cachés que contient le livre. Car l'ouvrage apparaît comme présentant une structure et une cohérence profondes, basées sur la mise en cohérence de la conception panthéiste de l'univers de l'auteur avec nos perspectives scientifiques actuelles les plus avancées en matière de cosmologie, de physique ainsi qu'avec les solutions de plusieurs problèmes philosophiques contemporains. Montrer de manière synthétique comment une vision panthéiste du monde peut se montrer compatible avec nos points de vue les plus récents en matière d'univers multiples, de physique et d'informatique quantique, de même qu'avec la théorie de la relativité et les discussions récentes relatives à l'omniscience, le problème du Mal, l'argument du bon réglage, les effets de sélection observationnels, etc., apparaît comme une entreprise à la fois immense et profondément originale.

On observera ici que Leslie est coutumier de ce type de travaux d'envergure. Il suffit pour cela de considérer son œuvre relative à l'argument de l'Apocalypse. Il 
faut évaluer ici la tâche immense qui consiste à défendre point par point l'argument de l'Apocalypse contre une bonne centaine d'objections différentes. Mais cette défense vigoureuse de l'argument de l'Apocalypse a permis en retour le développement d'une riche littérature, qui ne cesse d'éclairer nombre de domaines jusque-là ignorés.

La forme de panthéisme décrite par Leslie, inspirée de Spinoza, se caractérise par le fait que chacun d'entre nous n'est autre que la structure de pensées divines. Car seul existe l'esprit divin. Les galaxies, les planètes, les montagnes, les êtres humains que nous sommes, les animaux, les fleurs ne sont rien d'autre que des structures au sein de pensées divines. Ainsi que le fait remarquer Leslie, ceci est cohérent avec la façon dont les physiciens eux-mêmes décrivent les objets physiques, soit en spécifiant leurs propriétés intrinsèques. Toutefois, Leslie se démarque d'une conception panpsychiste où tous les êtres et les objets qui composent notre univers possèdent des propriétés mentales. Car selon l'auteur, les objets physiques tels que les arbres, les rochers, le sable existent en tant que structures au sein de l'esprit divin, mais sans être eux-mêmes dotés de conscience ou de pensée. Ici, toutes les choses ne sont pas dotées de conscience, mais elles sont cependant telles qu'une conscience de ces dernières existe.

De plus, des univers en nombre infini peuvent exister en tant que structures dans l'esprit divin. La théorie de l'auteur se révèle ainsi compatible avec les conceptions cosmologiques récentes basées sur l'existence d'univers multiples. L'un de ces univers est ainsi le nôtre, qui présente des caractéristiques et un réglage de ses paramètres (le rapport des masses respectives de l'électron et du proton, la charge de l'électron, la constante de gravitation, la constante de Planck, etc.) tels qu'il permet l'émergence d'une vie intelligente.

Plus encore, Leslie suggère l'existence non pas d'un esprit divin unique, mais bien d'une infinité d'esprits divins. Chacun est absolument identique aux autres, mais possède cependant une conscience autonome de sa propre existence.

Dans ce contexte, quel est donc le statut des objets abstraits tels que les entiers naturels? Selon certains philosophes, les objets abstraits constituent également des pensées divines. Tel est notamment le point de vue avancé par Alvin Plantinga, selon lequel les entiers naturels constituent des pensées divines. Mais Leslie adopte un point de vue différent, qui s'affranchit de l'existence des objets abstraits. Car ces derniers, de même que nos pensées, résultent de l'activité de nos cerveaux, qui sont eux-mêmes des structures au sein de l'esprit divin.

Leslie développe également le thème de l'omniscience. Pour Leslie, Dieu connaît simplement tout ce qui vaut la peine d'être connu (God knows everything worth knowing). Ceci apparait probablement plus plausible que l'idée selon laquelle Dieu possède toute connaissance, qui se heurte notamment à l'existence logiquement impossible, déjà notée par Patrick Grim, de l'ensemble qui contient absolument toutes les vérités. Notre conception préthéorique d'un Dieu omniscient pourrait bien se révéler naïve, ainsi que le souligne l'auteur, car nombre de faits insignifiants pourraient s'y révéler des connaissances indésirables.

Enfin, Leslie développe également le point de vue selon lequel Dieu existe par nécessité éthique (because of its ethical requiredness). L'existence de Dieu et du cosmos dans sa totalité est éthiquement nécessaire, de toute éternité. Cet argument pourrait aussi se révéler plus convaincant que certains arguments ontologiques. Car une telle nécessité éthique possède, selon Leslie, un pouvoir créateur. Mais un tel 
pouvoir créateur, d'essence platonicienne, ne procède d'aucune cause extérieure. Il est simplement inhérent par nature à la nécessité éthique.

L'ouvrage de Leslie manifeste également l'expression courageuse d'un point de vue. Car une telle conception panthéiste ne constitue pas un point de vue en vogue dans la philosophie analytique contemporaine. En outre, le panthéisme de Leslie constitue également une variante de panpsychisme. Mais l'attitude de l'auteur se révèle éminemment constructive, car elle nous contraint à nous pencher de manière plus attentive sur des doctrines que nous aurions tendance à rejeter trop facilement. On pourra adhérer ou non à la théorie panthéiste et panpsychiste décrite dans Infinite Minds. Mais pour la plupart des lecteurs, dont on peut penser qu'ils n'adhéreront pas à la variante de panpsychisme ainsi décrite, l'ouvrage de Leslie constitue néanmoins une synthèse admirable et hautement originale, montrant comment une construction étonnante peut être élaborée autour du modèle panthéiste, tout en apportant des réponses à de nombreux problèmes philosophiques contemporains. L'ouvrage procurera des arguments nouveaux aux défenseurs du panpsychisme. Mais Infinite Minds se révélera également indispensable aux détracteurs du panpsychisme, qui y trouveront une défense particulièrement forte et structurée.

PAUL FRANCESCHI

Université de Corse

\section{Michel Hulin, Śarikara et la non-dualité, Bayard, Paris, 2001, 278 p.}

Śankara est sans aucun doute le mieux connu des penseurs indiens, ce qui ne signifie pas qu'il le soit bien. D'abord, son œuvre est immense, et puis elle prend le plus souvent la forme de gros commentaires touffus, chargés de débats dont les enjeux échappent à celui qui ne s'est pas spécialisé en philosophie indienne. C’est pourquoi la connaissance que nous avons de sa pensée se limite habituellement à deux ou trois thèmes essentiels, à savoir que le monde est une illusion produite par la $m \bar{a} y \bar{a}$, qu'une seule chose est réelle, le Soi, et peut-être aussi que le salut vient du savoir. Ces thèses de Śankara séduisent par leur originalité, bien qu'elles ne s'accordent guère avec l'intuition. Car enfin, s'il n'y a de vérité que du Soi, comment expliquer que nous percevions le monde comme réel? Qu'est-ce qui nous permet de douter de sa réalité ? Et si le savoir empirique est d'emblée disqualifié comme portant sur une chose irréelle, quelle sorte de savoir Śankara recherche-t-il? Ce sont là quelques-unes des questions sur lesquelles Michel Hulin se penche dans son dernier livre.

L'ouvrage, qui comprend quatre parties, s'ouvre sur une présentation de la vie et de l'œuvre du philosophe non dualiste. M. Hulin identifie les différentes sources qui nous en parlent et évalue leur fiabilité. Il dépeint avec brio l'action de Śankara comme réformateur d'un hindouisme éclaté en une multitude de sectes, dont la diversité des pratiques ne connaissait pas de frein, en la situant dans le contexte social et culturel de l'Inde de la fin du viI ${ }^{\mathrm{e}}$ siècle (la tradition veut que Śankara soit né en 788 et mort en 820 , mais la recherche récente, dans laquelle M. Hulin s'inscrit, tend à montrer qu'il a eu une vie plus longue et que sa période d'activités est antérieure au milieu du viI ${ }^{\mathrm{e}}$ siècle). 